\title{
Evaluación del comportamiento de clones de Tectona grandis L. f. en suelos vertisoles de la Península de Nicoya, Costa Rica
}

\author{
Evaluation of the behavior of clones of Tectona grandis L. f. in vertisol soils of Nicoya \\ Peninsula, Costa Rica
}

\author{
Sara Molina-Quesada ${ }^{1}$ Carolina Alfaro ${ }^{1} \bullet$ Olman Murillo $^{2}$ (iD) $\bullet$ Yorleny Badilla $^{2}$ (iD) $\bullet$ Ricardo Luján ${ }^{3}$
}

\begin{abstract}
A Tectona grandis L. f. (Teak) genetic test consisting of 40 clones and one control (24 ramets per clone), distributed in a complete randomized block design, with six repetitions and two pairs of plants per clone per block (parcel), was evaluated. The trial was established by BARCA company and the "Cooperativa de Mejoramiento Genético Forestal" (GENFORES) in August 2015, nearby Jicaral, Nicoya Peninsula, Costa Rica. The soil order in the test site corresponds to a vertisol order, which is scarcely used for forest plantations due to its high percentage of expandable clays (type 2:1 montmorillonitic) that produce the formation of dynamic cracks during the dry season. At 8 months of age was conducted the first evaluation of this clonal test, where the collar diameter and total height of each individual was quantified, in addition to evaluating the condition of forking, presence of sprouts, survival and phytosanitary status of each plant. The collected data was analyzed through BLUP/REML procedures in SELEGEN software to obtain all genetic parameters. We obtained high results of mean clonal heritability in collar diameter $\left(h^{2} \mathrm{mc}=83 \%\right)$, total height $\left(h^{2} \mathrm{mc}=80 \%\right)$ and survival $\left(h^{2} \mathrm{mc}=74 \%\right)$. Accuracy of the genetic parameters registered values higher than 0.86 in all traits and parameters. At 8 month old genotypes 2, 9, 13, 21, 32 and 33 exhibited a tolerance tendency to vertisols soils. Meanwhile, clone 34 registered the worst adaptation in all clonal collection. The survival recorded was $84 \%$ that evidence the adaptation potential for some genotypes to this soil types in the region. Through selecting programs, it is possible to identify genetic materials able to adapt and produce acceptable production results in this type of soils.
\end{abstract}

Key words: Vertisol, genetic improvement, GENFORES, SELEGEN.

1. Estudiante, Escuela de Ingeniería Forestal, Tecnológico de Costa Rica; Cartago, Costa Rica;

molinaquesada.sara@gmail.com; caritoalro@gmail.com

2. Escuela de Ingeniería Forestal, Tecnológico de Costa Rica, Cartago, Costa Rica; olmuga@yahoo.es; yorlenybadilla@yahoo.es

3. Gerencia, Brinkman y Asociados Reforestadores

de Centro América S. A.; Puntarenas, Costa Rica; 


\section{Resumen}

Se evaluó un ensayo genético de Tectona grandis L. f. (Teca) conformado por 40 clones y un testigo (24 rametos por clon) distribuidos en un diseño de bloques completos al azar, con seis repeticiones y dos parejas de cada clon por bloque (parcela). El ensayo fue establecido por la empresa BARCA y la Cooperativa de Mejoramiento Genético Forestal (GENFORES) en agosto del año 2015, cerca de Jicaral, Península de Nicoya, Costa Rica. El suelo del sitio corresponde al orden vertisol, el cual es escasamente utilizado para plantaciones forestales debido a su alto porcentaje de arcillas expandibles (tipo 2:1 montmorillonítico), que forman grietas dinámicas durante el período seco. A los 8 meses de edad se realizó la primera evaluación del ensayo clonal, donde se cuantificó el diámetro a la base y altura total de cada individuo, además de evaluar la condición de bifurcación, rebrote, sobrevivencia y estado fitosanitario de cada planta. Los datos recopilados se analizaron mediante el procedimiento BLUP/REML del software SELEGEN para obtener los parámetros genéticos. Se obtuvieron resultados elevados de heredabilidad media del clon en diámetro a la base $\left(h^{2} \mathrm{mc}=83 \%\right)$, altura total $\left(h^{2} \mathrm{mc}=80 \%\right)$ y sobrevivencia $\left(h^{2} \mathrm{mc}=74 \%\right) . \mathrm{La}$ exactitud de los parámetros registró valores superiores al 0,86 en todos los caracteres y parámetros. A los 8 meses de edad los genotipos 2, 9, 13, 21, 32 y 33 exhibieron una clara tolerancia a estos suelos vertisoles. Mientras que el genotipo 34 registró el peor desempeño y menor adaptación de la colección de clones evaluada. La sobrevivencia registrada fue de un $84 \%$, lo que evidencia el gran potencial de adaptación de algunos genotipos a estas condiciones de suelo. A través de la selección genética es posible identificar materiales capaces de adecuarse y producir resultados aceptables de producción en suelos sonsocuites (vertisoles).

Palabras clave: Vertisol, mejoramiento genético, GENFORES, SELEGEN.

\section{Introducción}

La teca (Tectona grandis L. f.) es una de las especies maderables más conocidas a nivel mundial. Pertenece a la familia Lamiaceae y es originaria de la India, Myanmar, Tailandia e Indochina [1]. Su cultivo se extendió a regiones de Asia, África y América Central a principios del siglo XIX; sin embargo, fue hasta las últimas décadas de éste mismo periodo que se provocó un auge en su introducción al desarrollo económico [2].

La comercialización de madera para teca en Costa Rica a partir del año 2010 vivió un aumento en las exportaciones de madera en bruto, sin embargo, a partir del 2013 presentó una decaída a causa de la disponibilidad de otras especies como ciprés, melina y algunas de madera dura y semidura provenientes de bosque natural, a pesar de esto las exportaciones en el 2017 aumentaron a países como la India [3]. En Latinoamérica es la especie que mantiene mayor cantidad de área reforestada [4], en sitios de calidad media a buena [5], [6].

El crecimiento y desarrollo de la teca se ve afectado por las condiciones de sitio y variables climáticas; se ha reportado que la especie crece bien en sitios donde las precipitaciones superan los $1800 \mathrm{~mm}$ año-1, con velocidad de viento media y un periodo de 5 meses secos [7]; suelos fértiles de origen aluvial, moderadamente profundos (mayores a $90 \mathrm{~cm}$ ); con temperatura media y pendientes despreciables. Además, las investigaciones recomiendan evitar suelos mal drenados [6], [8].

En Costa Rica es posible encontrar diez de los 12 grandes órdenes de suelo reconocidos por los taxónomos, según Alvarado y Mata [6], exceptuando los Aridisoles (suelos desérticos) y Gelisoles (suelos congelados). La presencia de una alta variabilidad de materiales parentales en un relieve heterogéneo y sometidos a la acción de condiciones climáticas y biológicas particulares, han originado en el país, en un tiempo relativamente corto, esta gran variedad de suelos [9].

Los vertisoles son quizá, junto con unos pequeños parches de Mollisoles que existen asociados a ellos, los suelos más fértiles que existen en el país [9]. Se caracterizan por ser oscuros, con $\mathrm{pH}$ de neutro a básico. Poseen un alto porcentaje de arcillas que permiten la formación de grietas que se abren y cierran periódicamente dependiendo de la humedad y precipitación. En Costa Rica se conocen popularmente como "sonsocuites" y según Alvarado, Mata y Chinchilla [10], ocupan un área aproximada de $621 \mathrm{~km}^{2}(1,3 \%$ del territorio), encontrándose mayoritariamente en la región seca al noreste del Pacífico [6].

La abundancia de arcillas expandibles (del tipo 2:1 montmorillonítico) en los vertisoles, ocasiona serias dificultades en el movimiento vertical del agua [9], resultando prácticamente impermeables y anegados durante la mayor parte de la época lluviosa. Adicionalmente, estos suelos presentan alta cohesividad, adhesividad y plasticidad en la superficie; por lo tanto, pueden cambiar su forma en gran medida, quebrándose cuando se secan, y llegar a ser extremadamente resbaladizos y pegajosos cuando están mojados [11].

Los vertisoles están presentes en amplias zonas climáticas, desde el trópico húmedo hasta las zonas áridas [12], pero son más abundantes en las regiones tropicales y subáridas. En los trópicos ocupan el $60 \%$ 
del área total [12], donde se han utilizado, en su mayoría, para la agricultura inundada [13].

Este gran orden ha atraído la atención global en investigación, enfocándose principalmente en cambios que optimicen su uso [12]. Adicionar granza de arroz, cereales o leguminosas a los cultivos en suelos vertisoles son ejemplos de dichos estudios [14]. Sin embargo, se considera que los vertisoles son poco adecuados para la mayoría de las operaciones forestales [11] debido a que las raíces de los árboles no logran penetrar profundamente.

Cambiar las características físicas del suelo conlleva gran inversión económica; pero ¿por qué no plantar el genotipo adecuado? La investigación sobre el desarrollo en crecimiento y rendimiento de clones de teca en suelos vertisoles, es escasa.

La tolerancia a los suelos marginales podría tener un impacto económico importante en las plantaciones de teca. Es sabido que la teca es una especie que demanda suelos fértiles, lo que obliga a los inversionistas a buscar suelos ricos y de alta productividad, que hoy día son muy costosos y utilizados en cultivos de mayor rentabilidad y mejor flujo de caja [15]. En cualquier terreno de gran extensión, siempre hay sectores con áreas de suelos marginales no recomendables para el establecimiento de la teca.

Según Murillo et al. [16], la experiencia en teca señala que una de las características a mejorar por medio de investigación a nivel genético, es probar y seleccionar los mejores genotipos para el crecimiento en suelos marginales. El objetivo del presente trabajo fue evaluar el comportamiento de clones de teca en suelos con características vérticas.

\section{Materiales y métodos}

Durante el mes de agosto del año 2015 se estableció un ensayo genético clonal con 40 genotipos de la especie teca, además del testigo, en la Península de Nicoya, Puntarenas, Costa Rica. La temperatura media del sitio fluctúa entre los $26-28^{\circ} \mathrm{C}$ [17], la precipitación media anual corresponde a $1720 \mathrm{~mm}$ [18] y se reporta una temporada seca de entre los 4 y 6 meses cada año. Los suelos presentes en el ensayo corresponden al orden vertisoles, conocidos en nuestro país comúnmente como "sonsocuites", debido a su alta impermeabilidad y agrietamiento (figura 1). El sitio se encuentra en la zona de vida Bosque Húmedo Tropical [17] según la clasificación de Leslie Holdridge.

El ensayo genético clonal fue establecido por la Cooperativa de Mejoramiento Genético Forestal (GENFORES) bajo el diseño experimental desarrollado

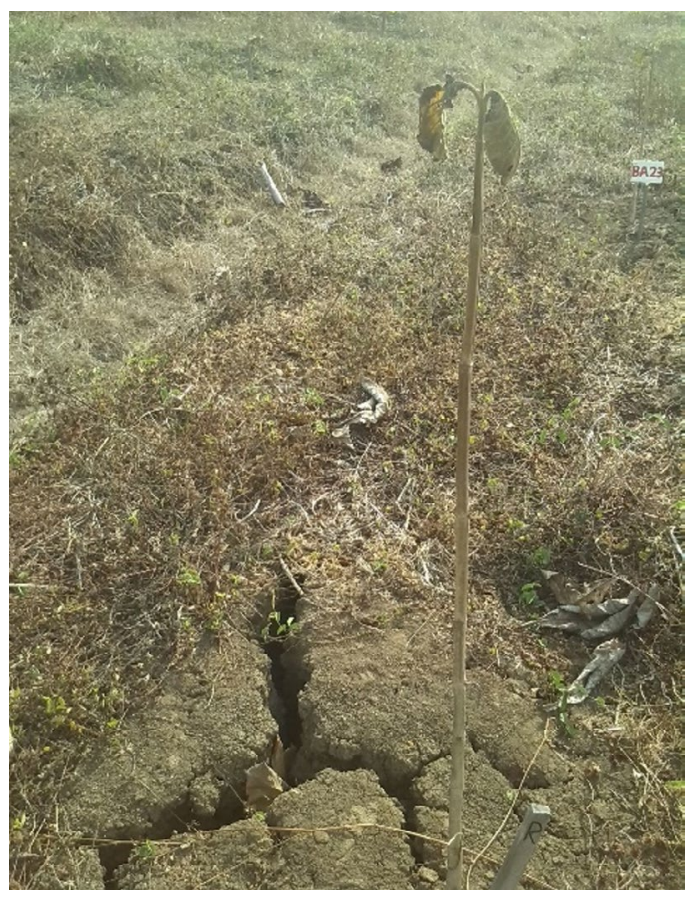

Figura 1. Agrietamiento característico de los suelos del orden vertisol, sitio del ensayo genético clonal en estudio, Península de Nicoya, Puntarenas, Costa Rica.

Figure 1. Characteristic cracking of vertisol soils in clonal genetic test study site, Nicoya Peninsula, Puntarenas, Costa Rica.

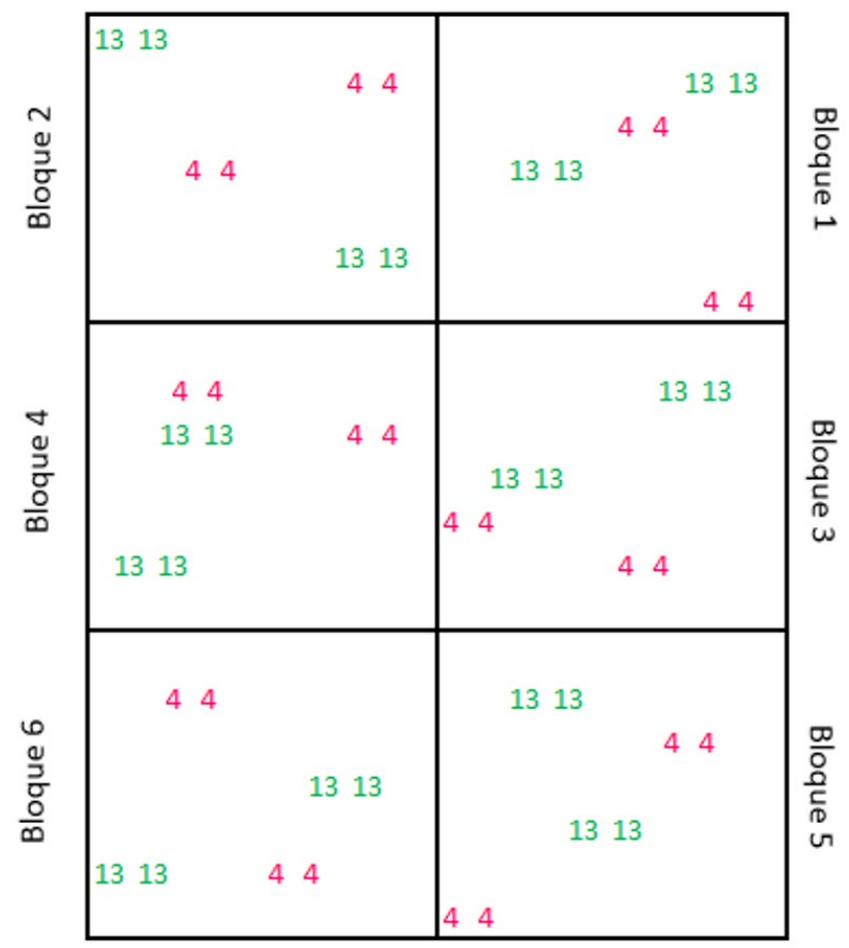

Figura 2. Diseño experimental desarrollado por [19] para la evaluación genética. Cada clon se evalúa en 2 parejas distribuidas aleatoriamente dentro de cada bloque.

Figure 2. Experimental design developed by [19] for genetic evaluation. Each clone is evaluated in 2 couples distributed randomly within each block. 


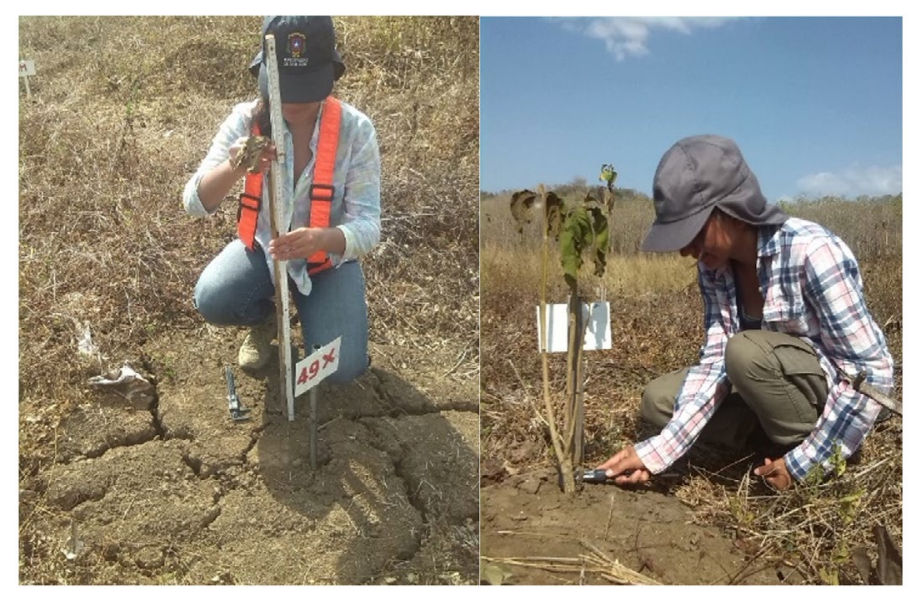

Figura 3. Proceso de evaluación del ensayo genético de Tectona grandis L. f. en suelos vertisoles, Península de Nicoya, Puntarenas, Costa Rica..

Figure 3. Evaluation process of the genetic test of Tectona grandis L. f. in vertisol soils, Nicoya Peninsula, Puntarenas, Costa Rica.

por [19], que consiste en un diseño de seis bloques completos al azar. Dentro de cada bloque se encuentran dos parejas de cada clon distribuidos de forma aleatoria, como se muestra en la figura 2. Cada bloque está conformado por $14 \times 12$ filas con dos hileras de borde y cuatro plantas de relleno para un total de 168 plantas/bloque. Los árboles fueron plantados a un distanciamiento de $4 \times 3 \mathrm{~m}$ y el sitio no tuvo ninguna preparación anterior al establecimiento.

En marzo del año 2016 (a los 8 meses de edad), se realizó la primera medición del ensayo genético clonal, con 40 clones (tratamientos) y un testigo (proveniente del huerto semillero del Centro Agrícola Cantonal de Hojancha$\mathrm{CACH}$ ). A cada individuo se le midieron las variables indicadoras de crecimiento: diámetro a la base $(\mathrm{cm})$ y altura total $(\mathrm{cm})$, utilizando el vernier electrónico y el metro respectivamente. Además, se evaluó la condición de bifurcación, rebrote, sobrevivencia y estado fitosanitario de cada planta (figura 3).

Los datos de campo fueron editados y ordenados en el programa de Microsoft Office, Excel, para su posterior evaluación. Se utilizó el software SELEGEN [20] para el análisis genético de la base de datos obtenida. Este programa se basa en el procedimiento de Máxima Verosimilitud Restringida (REML) y Mejor Predictor Lineal No Sesgada (BLUP), que permite optimizar la evaluación y selección genética, y establecer una jerarquía para cada carácter de importancia [20].

Se analizaron las diferencias genéticas entre clones, con el modelo 02: "Bloques completos al azar, prueba de clones no emparentados, varias plantas por parcela".
Seguidamente, se agruparon los clones de acuerdo con la empresa que aportó el material genético. De esta manera se obtuvieron 8 grupos que fueron tratados como procedencias y se analizaron bajo el modelo 24: "Bloques completos al azar, prueba de procedencias, varias plantas por parcela". A continuación, los modelos matemáticos que fueron utilizados por el software [21]:

$$
\begin{aligned}
& \text { Modelo 02: } y=X r+Z g+W p+e \\
& \text { Modelo 24: } y=X r+Z v+W p+e
\end{aligned}
$$

Donde "y" es el vector de datos, "r" es el vector de los efectos de repetición (asumidos como fijos) sumados a la media general, " $g$ " es el vector de los efectos genotípicos (asumidos como aleatorios), "v" es el vector de los efectos genotípicos de poblaciones (asumidos como aleatorios), "p" es el vector de los efectos de la parcela, y "e" es el vector del error de los residuos (aleatorios). Las letras mayúsculas representan las matrices de incidencia para dichos efectos.

Además, se analizó el ranking genético de cada variable, las correlaciones genéticas entre ellas y se cuantificó la sobrevivencia del ensayo genético por bloque y por clon.

\section{Resultados}

Se registraron valores de heredabilidad altos para las variables cuantitativas evaluadas: diámetro a la base $(84 \%)$ y altura total $(80 \%)$, así como también la sobrevivencia de cada clon, con $74 \%$ (cuadro 1). El crecimiento promedio de los individuos en general fue de $1,79 \mathrm{~cm}$ de diámetro a la base, lo que indica el engrosamiento de $0,22 \mathrm{~cm}$ por mes, aproximadamente, en estos primeros 8 meses. En cuanto a la altura total para todos los clones, se registra una tasa de crecimiento de $8,52 \mathrm{~cm} \mathrm{mes}^{-1}$.

En el cuadro 1 se observa que la varianza genética entre clones resulta muy amplia en la variable altura, amplia para el diámetro a la base y baja en cuanto a sobrevivencia. La heredabilidad individual ajustada por los efectos que produce la parcela se observa entre $12 \%$ y $25 \%$. Por otro lado, los efectos ambientales representan un porcentaje bajo, específicamente de $10 \%$ para el diámetro a la base, $6 \%$ para la altura total y $3 \%$ en relación con la sobrevivencia; lo que denota la poca variabilidad que se le atribuye al efecto ambiente dentro del análisis genético, parámetro que caracteriza la base de datos como robusta para el estudio.

Se observan resultados elevados de heredabilidad media del clon en diámetro a la base $\left(\mathrm{h}^{2} \mathrm{mc}=83 \%\right)$, altura total $\left(h^{2} \mathrm{mc}=80 \%\right)$ y sobrevivencia $\left(h^{2} \mathrm{mc}=74 \%\right)$; mientras 
Cuadro 1. Parámetros genéticos de 40 clones de Tectona grandis L. f. a los 8 meses de edad en suelos vertisoles, Península de Nicoya, Puntarenas, Costa Rica.

Table 1. Genetic parameters of 40 clones of Tectona grandis L. f. at 8 months of age in vertisol soils, Nicoya Peninsula, Puntarenas, Costa Rica.

\begin{tabular}{|c|c|c|c|}
\hline Parámetro & Diámetro a la base $(\mathrm{cm})$ & Altura total $(\mathrm{cm})$ & Sobrevivencia \\
\hline Vg & 0,13 & 259,72 & 0,02 \\
\hline Vparc & 0,06 & 99,34 & 0,01 \\
\hline Ve & 0,37 & 1137,47 & 0,11 \\
\hline $\mathrm{Vf}$ & 0,56 & 1496,53 & 0,13 \\
\hline$h^{2} g$ & $0,23 \pm 0,05$ & $0,17 \pm 0,04$ & $0,12 \pm 0,03$ \\
\hline$c^{2}$ parc & 0,10 & 0,07 & 0,03 \\
\hline $\mathrm{h}^{2} \mathrm{mc}$ & 0,83 & 0,80 & 0,74 \\
\hline Exactitud & 0,91 & 0,90 & 0,86 \\
\hline Cvgi\% & 19,87 & 23,64 & 6,80 \\
\hline Cve\% & 21,67 & 28,73 & 9,82 \\
\hline $\mathrm{CVr}$ & 0,92 & 0,82 & 0,69 \\
\hline PEV & 0,02 & 51,32 & 0,00 \\
\hline SEP & 0,15 & 7,16 & 0,06 \\
\hline Media general & 1,79 & 68,18 & - \\
\hline
\end{tabular}

$\mathrm{Vg}$ = varianza genotípica entre clones; Vparc = varianza ambiental entre parcelas en distintos bloques; Ve = varianza residual o no explicada por el modelo (ambiental); $\mathrm{Vf}=\mathrm{Vg}+\mathrm{Vparc}+\mathrm{Ve}=$ varianza fenotípica total; $\mathrm{h}^{2} \mathrm{~g}=$ heredabilidad individual en el sentido amplio, de los efectos genotípicos totales; $\mathrm{c}^{2}$ parc $=$ coeficiente de determinación de los efectos de la parcela (efecto ambiental); $\mathrm{h}^{2} \mathrm{mc}=$ heredabilidad media del clon asumiendo sobreviviencia completa; Exactitud = exactitud de los datos; CVgi $\%=$ coeficiente de variación genética aditiva individual; $\mathrm{CVe} \%=\mathrm{coeficiente}$ de variación experimental; $\mathrm{CVr}=$ coeficiente de variación relativa; $\mathrm{PEV}=$ varianza del error de predicción de valores genotípicos, asumiendo sobrevivencia completa; SEP = desviación estándar del valor genotípico predicho de clones, asumiendo sobrevivencia completa [22].

que la exactitud de los datos respalda su veracidad, con valores superiores al $86 \%$ en todas las variables (cuadro 2).

Las variables cualitativas analizadas, que en este caso indican el estado de vigor del árbol, se encuentran con buen índice. El 1,45\% de los individuos vivos presentó bifurcación, apenas el 0,60 \% de los árboles indicó un estado fitosanitario deficiente y el 2,06\% con presencia de rebrotes.

Como segundo resultado del análisis de datos se obtuvo la posición de cada clon en el ranking genético de diámetro a la base y altura total, como se muestra en el cuadro 2. Algunos clones cambian levemente de posición, pero mantienen su tendencia. El clon 2 encabeza la lista de ambas variables, seguido por el clon 1 y el 21 en diámetro y altura respectivamente. El 13 y clon 33 se ubican en tercer y quinto lugar del ordenamiento, posicionándose dentro de los mejores 5 clones para ambas variables. El clon 34 registró los peores crecimientos en las variables estudiadas, y en general, existe una tendencia marcada de algunos clones por posicionarse en los últimos lugares (clones con el código 40, 24, 19, 36, 38, 37).
Los clones 7 y 17 fueron aquellos que presentaron, con al menos un individuo, más de $2 \mathrm{~m}$ de altura a la edad de 8 meses. Por otro lado, el clon 1 fue el que presentó el individuo con el mayor diámetro de la base de datos obtenida en campo y como se muestra en el cuadro 2 , se encuentra en la segunda y treceava posición de los mejores genotipos para diámetro a la base y altura en el ensayo evaluado.

En las figuras 4 y 5 puede observarse que, en la población experimental, con tan solo ocho meses de edad, existen clones con tendencias de mayor crecimiento en cuanto al diámetro y la altura. Se muestra la distribución de mayor a menor (izquierda a derecha) crecimiento en diámetro a la base (figura 4) por clon, con límites de confianza superior e inferior. El clon 2 muestra crecimiento con diferencias significativas comparado con los clones: $34,24,40,40$, 36, T, 37 y 38 ; por otro lado, su crecimiento en diámetro es estadísticamente similar al resto de la población.

Clones que no presentan traslape en sus límites de confianza, son estadísticamente diferentes. Como se muestra en la figura 5, donde el clon 2 vuelve a aparecer en el primer lugar de crecimiento, pero esta vez con respecto a altura total, se puede observar su diferencia 
Cuadro 2. Ranking genético de 40 clones de Tectona grandis L. f. y el testigo a los 8 meses de edad en suelos vertisoles, con respecto al diámetro a la base y altura total, Península de Nicoya, Puntarenas, Costa Rica.

Table 2. Genetic ranking of 40 clones of Tectona grandis L. f. and the control at 8 months of age in vertisol soils, with respect to diameter to base and total height, Nicoya Peninsula, Puntarenas, Costa Rica.

\begin{tabular}{|c|c|c|}
\hline \multirow{2}{*}{ Orden } & \multicolumn{2}{|c|}{ Ranking } \\
\hline & diámetro a la base & altura tota \\
\hline 1 & 2 & 2 \\
\hline 2 & 1 & 21 \\
\hline 3 & 13 & 33 \\
\hline 4 & 9 & 23 \\
\hline 5 & 33 & 13 \\
\hline 6 & 32 & 10 \\
\hline 7 & 21 & 9 \\
\hline 8 & 29 & 17 \\
\hline 9 & 22 & 32 \\
\hline 10 & 7 & 25 \\
\hline 11 & 23 & 29 \\
\hline 12 & 17 & 7 \\
\hline 13 & 10 & 1 \\
\hline 14 & 27 & 22 \\
\hline 15 & 11 & 6 \\
\hline 16 & 6 & 11 \\
\hline 17 & 4 & 16 \\
\hline 18 & 25 & 8 \\
\hline 19 & 31 & 26 \\
\hline 20 & 35 & 5 \\
\hline 21 & 16 & 27 \\
\hline 22 & 30 & 28 \\
\hline 23 & 26 & 4 \\
\hline 24 & 18 & 3 \\
\hline 25 & 15 & 35 \\
\hline 26 & 28 & 30 \\
\hline 27 & 12 & 18 \\
\hline 28 & 14 & 14 \\
\hline 29 & 3 & 31 \\
\hline 30 & 8 & 15 \\
\hline 31 & 20 & 39 \\
\hline 32 & 5 & 12 \\
\hline 33 & 39 & 20 \\
\hline 34 & 38 & 37 \\
\hline 35 & 37 & $\mathrm{~T}$ \\
\hline 36 & $\mathrm{~T}$ & 38 \\
\hline 37 & 36 & 19 \\
\hline 38 & 19 & 36 \\
\hline 39 & 40 & 24 \\
\hline 40 & 24 & 40 \\
\hline 41 & 34 & 34 \\
\hline
\end{tabular}

significativa con los siete clones posicionados más a la derecha del gráfico.

Además, se muestra en ambas figuras la media de la población en general y la media de los mejores 10 clones para ambas variables. Sin duda alguna existe gran diferencia entre ellas, mostrando que los clones 2 , 1 y 13 sobrepasan ambos promedios de diámetro a la base; y el clon 2, 22 y 33 los de altura total. Se posiciona tentativamente de esta manera el clon 2 como el mejor en cuanto a características cuantitativas de crecimiento en suelos vertisoles a los primeros 8 meses de edad, en la localidad de estudio.

En contraposición, existe un grupo de 7 clones, que ya manifiestan significativamente un crecimiento inferior al promedio general de diámetro $(1,79 \mathrm{~cm})$ y altura $(68,18$ $\mathrm{cm})$. Es importante mencionar que cuatro clones de este grupo tienen su origen en el lugar de estudio. Lo anterior agrega peso a la investigación realizada, ya que los clones en las mejores posiciones del ranking de crecimiento (Tabla 2), son procedentes de otros lugares del país diferentes a la Península de Nicoya, Puntarenas.

Los clones aportados por cada empresa (ocho en total) fueron agrupados, de manera que se comportaran como una misma procedencia. En las figuras 6 y 7 se muestra el ranking de las agrupaciones junto con el posicionamiento del testigo, para diámetro a la base y altura total del ensayo en suelos vertisoles.

En el cuadro 3 se presenta la matriz de correlaciones entre las variables que fueron evaluadas. Existe una relación bastante estrecha entre el diámetro a la base que tienen los individuos con respecto a su altura. Lo anterior respalda los resultados del ranking (cuadro 2), donde se aprecia la tendencia al desarrollo que tiene cada clon bajo las condiciones evaluadas. Por otro lado, la sobrevivencia se correlaciona con las variables cuantitativas, como es de esperarse: a mayor diámetro y/o altura, menor posibilidad de morir presenta el individuo (cuadro 3).

Se registró $84 \%$ de sobrevivencia en la totalidad del ensayo clonal, a los 8 meses de edad, en suelos vertisoles, de manera que la base de datos se considera altamente aceptable para analizar genéticamente.

El análisis de sobrevivencia por procedencia muestra resultados desde el $100 \%$ hasta el $42 \%$, como se observa en el cuadro 4. Los diez clones con mayor porcentaje de sobrevivencia presentan un promedio de diámetro mayor al promedio general $(1,79 \mathrm{~cm})$. Los clones que presentan alta mortalidad (clon 34 y 19), ambos con apenas un 42 $\%$, coinciden con las últimas posiciones en el ranking genético de las variables evaluadas. 


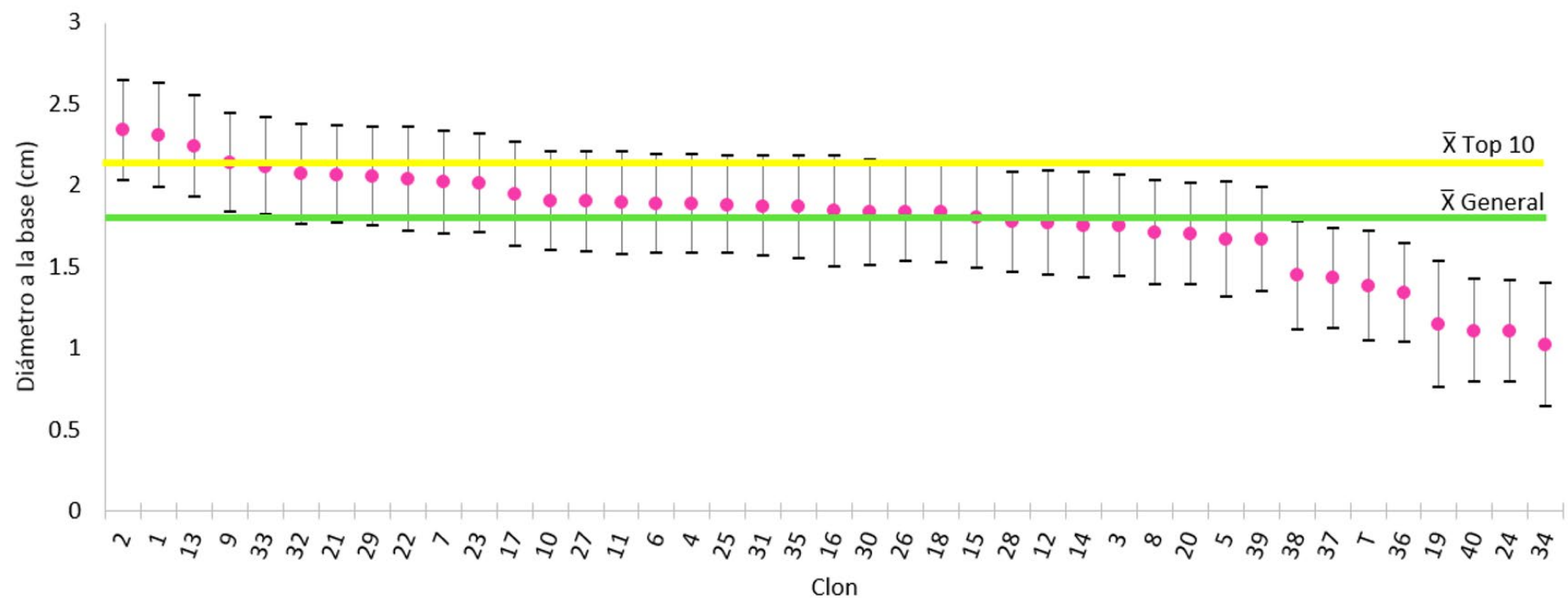

Figura 4. Valores genéticos y límites de confianza para el diámetro a la base de diferentes clones de Tectona grandis L. f. en suelos vertisoles, Península de Nicoya, Puntarenas, Costa Rica.

Figure 4. Genetic values and confidence limits for the diameter at the base of different clones of Tectona grandis L. f. in vertisol soils, Nicoya Peninsula, Puntarenas, Costa Rica.

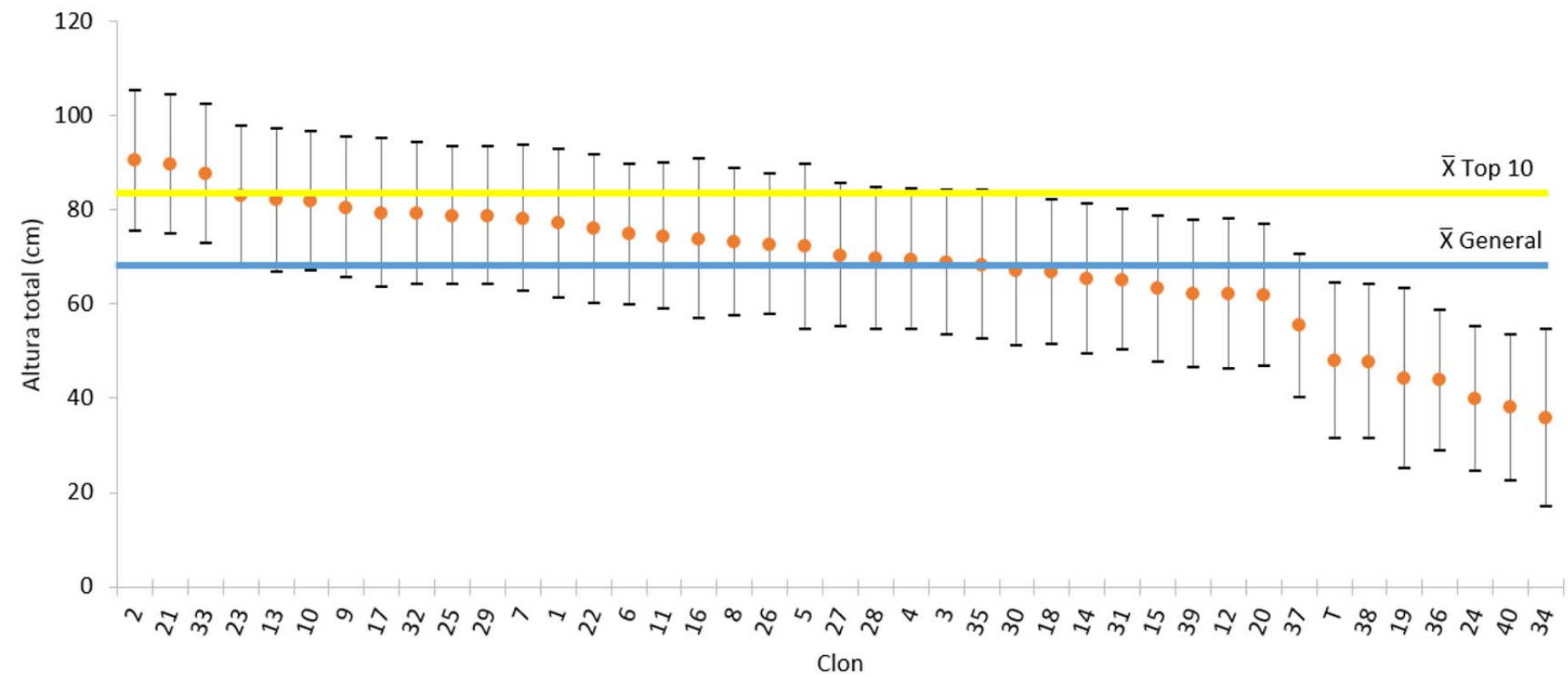

Fıgura 5. Valores genetıcos y limıtes de contıanza para la altura total de diterentes clones de Iectona grandıs L. t. en suelos vertısoles, Peninsula de Nicoya, Puntarenas, Costa Rica.

Figure 5. Genetic values and confidence limits for the total height of different clones of Tectona grandis L. f. in vertisol soils, Nicoya Peninsula, Puntarenas, Costa Rica.

La sobrevivencia mantiene valores similares en los distintos bloques, como se muestra en el cuadro 5. La poca variabilidad que presentan los datos verifica que el diseño de bloques se hizo de la manera correcta.

El factor procedencia establece una gran diferencia en cuanto a la sobrevivencia, con un distanciamiento del
$28 \%$ entre la procedencia con mayor y menor porcentaje de sobrevivencia. Como se muestra en el cuadro 6, los clones de la procedencia $C$ presentaron la mayor sobrevivencia, con los clones $6,7,21,26$ y 29; que se ubican en los lugares intermedios del ranking genético (cuadro 2). Como se esperaría, el testigo obtuvo menor número de individuos vivos. 


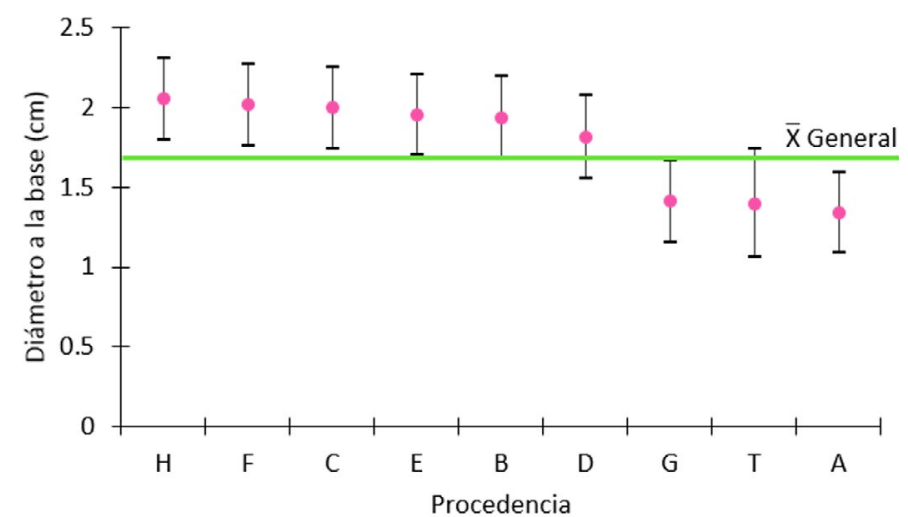

Figura 6. Valores genéticos y límites de confianza para el diámetro a la base y altura total de los clones de Tectona grandis L. f. agrupados en procedencias más el testigo (T), a los 8 meses de edad, en suelos vertisoles, Península de Nicoya, Puntarenas, Costa Rica.

Figure 6. Genetic values and confidence limits for the total height of different clones of Tectona grandis L. f. in vertisol soils, Nicoya Peninsula, Puntarenas, Costa Rica.

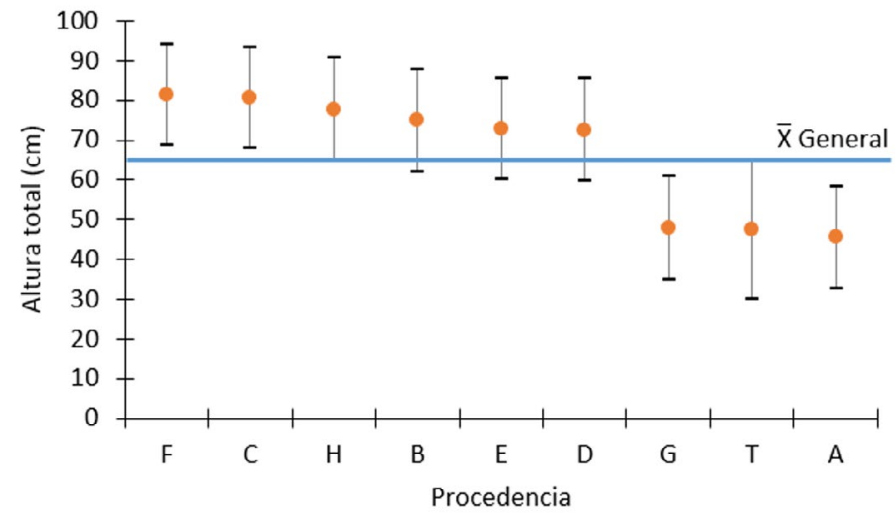

Figura 7. Valores genéticos y límites de confianza para altura total de los clones de Tectona grandis L. f. agrupados en procedencias más el testigo (T), a los 8 meses de edad, en suelos vertisoles, Península de Nicoya, Puntarenas, Costa Rica.

Figure 7. Genetic values and confidence limits for total height of the clones of Tectona grandis L. f. grouped in provenances plus the control (T), at 8 months of age, in vertisol soils, Nicoya Peninsula, Puntarenas, Costa Rica.

Cuadro 3. Matriz de correlaciones genéticas de las variables medidas en el ensayo genético de clones individuales de Tectona grandis L. f. ubicado en suelos vertisoles, Península de Nicoya, Puntarenas, Costa Rica.

Table 3. Matrix of genetic correlations of the variables measured in the genetic test of individual clones of Tectona grandis L. f. located in vertisol soils, Nicoya Peninsula, Puntarenas, Costa Rica.

\section{Altura total}

Sobrevivencia

Diámetro a la base $\quad 0,94^{\star \star \star}$

$0,52^{\star \star}$

Altura total

$0,51^{\star *}$
Cuadro 4. Clones de Tectona grandis L. f. con mayor y menor porcentaje de sobrevivencia a los 8 meses de edad en suelos vertisoles, Península de Nicoya, Puntarenas, Costa Rica.

Table 4. Clones of Tectona grandis L. f. with higher and lower percentage of survival at 8 months of age in vertisol soils, Nicoya Peninsula, Puntarenas, Costa Rica.

\begin{tabular}{|cc|}
\hline Clon & Sobrevivencia (\%) \\
\hline 10 & 100 \\
\hline 21 & 100 \\
\hline 25 & 100 \\
\hline 29 & 100 \\
\hline 33 & 100 \\
\hline 2 & 96 \\
\hline 4 & 96 \\
\hline 6 & 96 \\
\hline 9 & 96 \\
\hline 23 & 96 \\
\hline 39 & 79 \\
\hline 12 & 75 \\
\hline 14 & 75 \\
\hline 30 & 75 \\
\hline 38 & 71 \\
\hline 41 & 67 \\
\hline 16 & 63 \\
\hline 5 & 54 \\
\hline 19 & 42 \\
\hline & 42 \\
\hline 34 & \\
\hline
\end{tabular}

Cuadro 5. Porcentaje de sobrevivencia por bloque del ensayo genético de Tectona grandis L. f. a los 8 meses de edad en suelos vertisoles, Península de Nicoya, Puntarenas, Costa Rica.

Table 5. Percentage of survival per block of the genetic test of Tectona grandis L. f. at 8 months of age in vertisol soils, Nicoya Peninsula, Puntarenas, Costa Rica.

\begin{tabular}{|cc|}
\hline Bloque & Sobrevivencia (\%) \\
\hline I & 84 \\
\hline II & 85 \\
II & 85 \\
V & 84 \\
VI & 88 \\
\hline
\end{tabular}


Cuadro 6. Matriz de correlaciones genéticas de las variables medidas en el ensayo genético de clones individuales de Tectona grandis L. f. ubicado en suelos vertisoles, Península de Nicoya, Puntarenas, Costa Rica.

Table 6. Matrix of genetic correlations of the variables measured in the genetic test of individual clones of Tectona grandis L. f. located in vertisol soils, Nicoya Peninsula, Puntarenas, Costa Rica.

\begin{tabular}{|cc|}
\hline Procedencia & Sobrevivencia (\%) \\
\hline C & 95 \\
\hline E & 89 \\
\hline F & 89 \\
\hline B & 86 \\
\hline A & 84 \\
\hline H & 83 \\
\hline D & 81 \\
\hline G & 70 \\
\hline T & 67 \\
\hline
\end{tabular}

\section{Discusión}

\section{Evaluacion IAF}

El análisis de heredabilidad muestra valores sumamente altos para las variables diámetro a la base $\left(h^{2} \mathrm{mc}=83\right.$ $\%)$ y altura total $\left(h^{2} \mathrm{mc}=80 \%\right)$, esto a pesar de que el ensayo fue establecido con apenas 8 meses de anterioridad (cuadro 1), estos valores se encuentran muy por encima de los reportados por Narayanan et al. [22] quienes obtuvieron valores de heredabilidad para diámetro y altura de 34,3 y $19,4 \%$ respectivamente, valores similares son reportados por Espitia et al. [23] en Colombia, con una heredabilidad para la variable diámetro de $20 \%$ y para la altura de $25 \%$. Por lo tanto, es evidente el gran potencial de mejoramiento genético en suelos vertisoles en favor de individuos con mayor tolerancia y que probablemente, en el tiempo, ampliarán el rango de diferencia en respuesta al factor ambiente entre clones, siempre con la misma tendencia (figuras 4 y 5). Se espera entonces que la utilización en campo de clones genéticamente tolerantes a las características vérticas del suelo, contribuya de manera real y efectiva en la reducción de áreas desprovistas de reforestación.

El porcentaje de exactitud de los datos mostrado para cada variable (cuadro 1) respalda la calidad de base de datos que se utilizó para el análisis $(>0,86)$, lo que garantiza una buena estimación de todos los parámetros genéticos. [24] muestran un análisis genético con valores de exactitud mayores a 0,63 para Acacia mangium de cuatro años de edad; mientras que Salas-Rodríguez et al. [25] definen para tres de sus sitios de estudio indicadores de exactitud con valores superiores a 0,76 para la especie Gmelina arborea.

Los valores de los coeficientes de variación genética individual y del bloque son también bastante altos (superiores al $10 \%$ en la mayoría de los casos), lo cual es consistente y concordante con valores de heredabilidad altos.

El ordenamiento de los clones con base en su desarrollo de altura y diámetro (cuadro 2), fue visualmente eficiente para comprender el posicionamiento de los genotipos y verificar que los de mayor crecimiento están representados por ambas variables. Se sigue un patrón donde cada genotipo no se aleja más de doce posicionamientos entre los resultados del ranking. Además, existe gran consistencia en los primeros diez lugares de ordenamiento genético, donde el clon 2 muestra los mejores resultados en cuanto a rendimiento y crecimiento en suelos vertisoles.

En el grupo de los mejores 10 clones hay materiales de cinco empresas distintas. Además, los gráficos de las figuras 6 y 7 muestran que esas mismas cinco procedencias (significativamente tres de ellas) están por encima del promedio general en cuanto a diámetro a la base y altura total de los individuos. Las procedencias $\mathrm{H}$, $\mathrm{F}$ y $\mathrm{C}$ aparecen siempre en estos tres primeros puestos de desarrollo, sin embargo, no hay diferencias entre los límites de confianza de estas. El comportamiento que refleja mayor relevancia es el de las tres procedencias con crecimiento más bajo, donde el testigo $(T), G$, y $A$ se comportan de manera similar, reflejando el lento desarrollo en estos suelos.

El análisis genético registró una alta estabilidad entre el posicionamiento de los clones en relación con el diámetro a la base y con la altura total (cuadros 2 y 3), además de una alta correlación genética entre ambas $(0,94)$, como usualmente ocurre en especies forestales [23].

En cuanto a la sobrevivencia, se encontró que la correlación genética es recíprocamente proporcional a las características cuantificadas $(>0,51)$, así como los autores [19], definen correlaciones mayores a 0,41 en Dipteryx panamensis. Sin embargo, no es constante que los 10 mejores clones del ranking genético indiquen sobreviviencia completa (cuadro 4). Por otro lado, el cuadro 6 presenta el porcentaje de sobrevivencia por procedencia, donde se encuentra una tendencia regular en cantidad de individuos vivos. Esto respalda los datos del estudio, pues cada empresa fue analizada con cantidades similares de material genético vivo, con menor cantidad representada por la procedencia testigo (T). En el cuadro 5 se muestra que no existe diferencia marcada entre bloques, lo que optimiza el método de estratificación del estudio. De esta manera se comprueba que los resultados analizados con tan sólo ocho meses 
de edad del ensayo son mayoritariamente consecuencia del material genético establecido en condiciones de suelo con características vérticas.

Se recomienda hacer mediciones posteriores para monitorear el crecimiento de los clones que han obtenido los mejores resultados. Kumar [26] menciona que dos o tres años luego de plantar es aún una etapa relativamente temprana para obtener resultados definitivos, de manera que, para lograr mayor exactitud y seguridad, deben realizarse evaluaciones en años posteriores. Además, [27] recomiendan hacer una segunda evaluación para constatar que los clones seleccionados fueron consistentes en el tiempo.

\section{Conclusiones}

Se obtuvieron resultados altos de heredabilidad media del clon en diámetro a la base $\left(\mathrm{h}^{2} \mathrm{mc}=83 \%\right)$, altura total $\left(h^{2} \mathrm{mc}=80 \%\right)$ y sobrevivencia $\left(\mathrm{h}^{2} \mathrm{mc}=74 \%\right)$; mientras que la exactitud de los datos respalda su veracidad y confiabilidad, con valores superiores al $86 \%$ en todos los parámetros genéticos.

Los clones 2, 9, 13, 21, 32 y 33, exhibieron una clara tendencia de mayor crecimiento a estos suelos vertisoles.

El análisis de los clones por su procedencia (origen por empresa) determinó diferencias significativas, donde las colecciones con código $\mathrm{G}, \mathrm{A}$ y el testigo $(\mathrm{T})$, registraron el peor desempeño.

La sobrevivencia del $84 \%$ a los 8 meses del ensayo fue mejor de lo esperado, y evidencia el potencial de adaptación a estos suelos de algunos genotipos.

A través de la selección genética es posible desarrollar materiales capaces de adaptarse y producir resultados aceptables de producción en suelos sonsocuites (vertisoles).

\section{Referencias}

[1] P. Weaver, Tectona grandis L. f., Washington, D.C.: International Institute of Tropical Forestry. SO-ITF-SM-64, 1993, pp. 1-18.

[2] R. de Camino y J. Pierre, Las plantaciones de teca en América Latina: mitos y realidades, Turrialba: Centro Agronómico Tropical de Investigación y Enseñanza., 2013.

[3] A. Barrantes y S. Ugalde, "Balanza comercial y principales tendencias de las exportaciones e importaciones de madera y muebles de madera en Costa Rica". 2017. [Online]. Disponible en: https://www.onfcr.org/media/uploads/ documents/balanza-comercial-2017.pdf. [Accesado:Junio 1, 2018].

[4] H. Martínez, Teca (Tectona grandis L. f.): condiciones para su cultivo Fomento de la reforestación comercial para la mejora y conservación de las reservas de carbono, San José: Fondo Nacional de Financiamiento Forestal, 2015.

[5] W. Ladrach, " Manejo de plantaciones de la teca para productos sólidos", in Convención Nacional de la SAF (Society of American Foresters), Florida, 2009.

[6] A. Alvarado and R. Mata, Soils of Costa Rica: An Agroecological Approach, Chicago: The University of Chicago Press., 2015.

[7] A. Alvarado and B. Herrera, Nutrición y fertilización forestal en regiones tropicales, San José: Asociación Costarricense de la Ciencia, 2012

[8] A. Alvarado and J. Raigosa, Nutrición y fertilización forestal en regiones tropicales, San José: Asociación Costarricense de la Ciencia del Suelo. , 2012.

[9] F. Bertsch, A. Alvarado, C. Henriquez and R. Mata "Properties, geographic distribution, and management of major soil orders of Costa Rica", en Quantifying Sustainable Development The future of tropical economies, C, Hall, C. Leon and G. Leclerc. USA: Academic Press, 2000.

[10] A. Alvarado, R. Mata and M. Chinchilla, "Arcillas identificadas en suelos de Costa Rica a nivel generalizado durante el periodo 1931-2014: II. Mineralogía de arcillas en suelos con características vérticas y oxídico caoliníticas," Agronomía Costarricense, vol. 38, no. 1, pp. 107-131, 2014.

[11] C. Henríquez, G. Cabalceta, F. Bertsch and A. Alvarado, "Principales suelos de Costa Rica. Ministerio de Agricultura y Ganadería (MAG)," 2014. [Online]. Disponible en: http:// www.mag.go.cr/bibioteca_virtual_ciencia/suelos-cr.html. [Accesado Mayo 1, 2018].

[12] D. Pal, S. Wani and K. Sahrawat, "Vertisols of tropical Indian environments: pedology and edaphology," Geoderma, vol. 28, pp. 189-190, 2012.

[13] T. Araya, J. Nyssen, B. Govaerts, J. Deckers and W. Cornelis, "Impacts of conservation agriculture-based farming systems on optimizing seasonal rainfall partitioning and productivity on vertisols in the Ethiopian drylands," Soil \& Tillage Reseach, no. 148, pp. 1-13, 2015.

[14] N. Hulugalle, T. Weaver and L. Finlay, "Soil structure in permanent beds under irrigated cotton-based cropping systems in a Vertisol," Soil \& Tillage Research, no. 165, pp. 107-112, 2017.

[15] O. Murillo, J. Wright, O. Monteuuis and F. Montenegro, Mejoramiento genético de la teca en América Latina, Turrialba: CATIE, 2013.

[16] O. Murillo, M. Espitia and C. Castillo, Fuentes Semilleras para la Producción Forestal, Bogotá: Domar S.A.S., 2012.

[17] E. Ortiz and C. Soto, Atlas Digital de Costa Rica. Versión portable], Cartago: Instituto Tecnológico de Costa Rica., 2014.

[18] Instituto Meteorológico Nacional, "Datos climáticos, geoportal," 2016. [Online]. Disponible en: https://www.imn. ac.cr/web/imn/inicio. [Accesado: Mayo 1, 2016]. 
[19] V. Martínez-Albán, L. Fallas-Valverde, O. Murillo-Gamboa y Y. Badilla-Valverde, "Potencial del mejoramiento genético en Dipteryx panamensis a los 33 meses de edad en San Carlos, Costa Rica", Revista Forestal Mesoamericana kurú, vol. 13, no. 30, pp. 5-11, 2016.

[20] M. Resende, SELEGEN-REML/BLUP, Sistema Estatistico e Selecao Genetica Computadorizada, 2008.

[21] I. Pastrana-Vargas, M. Espitia-Camacho y O. MurilloGamboa, "Evaluación del potencial de mejoramiento genético en el crecimiento en altura de Acacia mangium Willd," Acta Agronómica, vol. 61, no. 2, pp. 143-150, 2012.

[22] M. Resende, "O Software Selegen-Reml/Blup," Documentos EMBRAPA., Campo Grande, Brasil: , 2006.

[23] C. Narayanan, P. Chawhaan and A. Mandal, "Inheritance Pattern of Growth and Wood Traits in Teak (Tectona grandis L.f.)," Silvae Genetica, vol. 58, no. 3, pp. 97-101, 2009.

[24] M. Espitia, O. Murillo y C. Castillo, "Ganancia genética esperada en teca (Tectona grandis L.f.) en Cordoba (Colombia)," Colombia Forestal, vol. 14, no. 1, pp. 81-93, 2010.

[25] B. Pavlotzky-Blank y O. Murillo-Gamboa, "Ganancia genética en Acacia mangium en Los Chiles, Zona Norte de Costa Rica," Agronomía Mesoamericana, vol. 23, no. 1, pp. 1-13, 2012.

[26] A. Salas-Rodríguez, O. Murillo-Gamboa, R. Murillo-Cruz y C. Ávila-Arias, " Evidencia de tolerancia genética a la pudrición del tronco en clones de Gmelina arborea Roxb. en Costa Rica," Revista Forestal Mesoamericana kurú, vol. 13, no. 32, pp. 30-39, 2016.

[27] A. Kumar, "Growth performance and variability in different clones of Gmelina arborea (Roxb.)," Silvae Genetica, no. 56, pp. , 56, 32-36, 2012.

\section{Este artículo debe citarse como:}

Molina-Quesada, S., Alfaro, C., Murillo, O., Badilla, Y., \& Luján, R. (2018). Evaluación del comportamiento de clones de Tectona grandis L. f. en suelos vertisoles de la Península de Nicoya, Costa Rica. Revista Forestal Mesoamericana Kurú, 16(38), 24-34. Doi. 10.18845/rfmk. v16i38.3993 